\title{
News from ASCO 2017
}

\author{
Chair: $\quad$ Marcus Schmidt ${ }^{\mathrm{a}}$ \\ Participants: Tanja Fehm ${ }^{b}$ Cornelia Liedtke ${ }^{c}$ Volkmar Müller $^{d}$ \\ ${ }^{a}$ Klinik und Poliklinik für Geburtshilfe und Frauengesundheit, Universitätsmedizin der Johannes Gutenberg-Universität Mainz, Mainz, \\ Germany; \\ ${ }^{b}$ Frauenklinik, Universitätsklinikum Düsseldorf, Düsseldorf, Germany; \\ ${ }^{c}$ Gynäkologie mit Brustzentrum, Charité-Universitätsmedizin Berlin, Berlin, Germany; \\ ${ }^{\mathrm{d}}$ Klinik und Poliklinik für Gynäkologie, Universitätsklinikum Hamburg-Eppendorf, Hamburg, Germany
}

\section{Question 1: What Were Your Highlights of This Year's ASCO?}

Fehm: One of the highlights of the ASCO meeting was the presentation of the results of the OlympiaD trial. This trial focused on the role of Olaparib in anthracycline and taxane pretreated, BRCA1/2-positive patients with metastatic disease. The other highlight was the APHINITY trial which investigates the role of the dual blockade in early HER2-positive breast cancer. The results have been eagerly awaited.

Liedtke: In my view, this year's ASCO's highlight was the presentation of the results of the OlympiaD study. Robson and colleagues presented the results of the primary endpoint analysis of progression-free survival (PFS) of a comparison of olaparib vs. monochemotherapy of physician's choice among patients with metastatic hereditary breast cancer. Inclusion criteria comprised patients with HER2-negative metastatic breast cancer with a deleterious or suspected deleterious genomic BRCA mutation, prior anthracycline and taxane exposure, and $\leq 2$ prior chemotherapy lines in the metastatic setting. In case of prior treatment with carboplatin, either no evidence of progression during treatment in the advanced setting or an interval of $\geq 12$ months since (neo)adjuvant treatment was necessary. The authors demonstrated a significant and clinically relevant benefit of the non-chemotherapy containing olaparib arm vs. chemotherapy with vinorelbine, capecitabine, or eribulin (median PFS 7.0 vs. 4.2 months, HR 0.58 (95\%CI $0.43-$ $0.80), \mathrm{p}=0.0009)$. Of note, response dynamics with respect to olaparib therapy were similar to those observed in association with chemotherapy, i.e. a median time to response of 47 vs. 45 days was noted, respectively. Subgroup analyses suggest that the benefit observed with respect to olaparib therapy was probably driven by a particular efficacy of olaparib among patients with triple-negative disease without prior platinum exposure. No new safety signal was observed. These analyses demonstrate that olaparib represents a valuable treatment option among patients with metastatic hereditary breast cancer, provided that olaparib be licensed in this indication in the future.

Müller: The positive results of the OlympiaD study were presented as only breast cancer abstract in the general plenary session and that should finally open the way of PARP inhibition for BRCAmutated patients and maybe also other patients with homologous recombination deficient tumors. The development of other PARP inhibitors might be also accelerated by this positive study.

\section{Question 2: Is Immunotherapy with Check-Point Inhibitors Hype or Hope in Breast Cancer?}

Fehm: Both. Several studies have been presented at the ASCO meeting investigating the efficacy of PDL-1 / PD antibodies as part of palliative treatment. The response rates were low, ranging from 5 to $25 \%$ depending on the definition of treatment benefit and pretreatment. Nevertheless, a subset of these patients responds well and is still under investigational drug treatment. Therefore, the major challenge will be to find valid biomarkers to identify those patients who will derive benefit from checkpoint inhibitors. In addition, we have to develop strategies to enhance the efficacy of immunotherapy, e.g. by adding radiotherapy or cytotoxic treatment. This will increase antigen release of tumor cells and may boost the immune system.

Liedtke: There is a growing body of evidence that checkpoint inhibition may become an important treatment option among patients with (particularly triple-negative) breast cancer. Although

\section{KARGER}

(c) 2017 S. Karger GmbH, Freiburg

Fax +497614520714
Prof. Dr. Marcus Schmidt

Abteilung für Konservative und Molekulare Gynäkologische Onkologie

Klinik und Poliklinik für Geburtshilfe und Frauengesundheit, Universitätsmedizin der Johannes Guten-

berg-Universität Mainz

Langenbeckstr. 1, 55131 Mainz, Germany

Marcus.Schmidt@unimedizin-mainz.de 
we do not yet have results from a phase III registration trial, there is an increasing understanding how this new therapy works. Based upon results presented at ASCO we assume that checkpoint inhibition is more effective the earlier it is used in the course of the disease. Furthermore, although monotherapy is efficacious to some extent we have seen higher response rates with monochemotherapy and even more with combination chemotherapy.

Müller: Although we all are excited about this new approach that seems to provide long-term benefit for some patients, I believe it is still not clear what will be the role in breast cancer and which patients could benefit from this. Therefore, we have to wait for further trial results.

\section{Question 3: Do We Have to Re-Write the Textbook of HER2-Positive Early Breast Cancer?}

Fehm: In same way, yes. De-escalation of systemic treatment in HER2-positive patients is an important issue. Results from the SHORT HER2 trial suggest that trastuzumab for 9 weeks may be nearly as effective as trastuzumab for 1 year in patients with small tumors and negative lymph nodes. Therefore, if patients have to stop trastuzumab for whatever reason (e.g. cardiotoxicity), they will still have a great benefit even from a shortened trastuzumab treatment. The updated results from the APT trial confirmed that 12 weeks of paclitaxel in combination with 1 year trastuzumab is an effective treatment in patients with small HER2-positive tumors (tumors $<2 \mathrm{~cm}$ ). In contrast, escalation of HER 2 treatment by adding pertuzumab to the current standard may add clinical benefit in ER negative and / or node positive patients as suggested by the APHINITY trial.

Liedtke: I assume that this question refers to the presentation of the primary endpoint data from the APHINITY study. The study randomized patients with HER2-positive early breast cancer (EBC) to chemotherapy plus trastuzumab plus placebo versus chemotherapy plus trastuzumab plus pertuzumab. Results were presented by Gunter von Minckwitz and showed that the addition of pertuzumab did result in a significant increase in 3-year disease-free survival (DFS) (94.1 vs. 93.2\%, HR 0.81 (95\%CI 0.66-1.00), p = 0.045). However, with an absolute difference of about $1 \%$ and a numberneeded-to-treat (NNT) to prevent 1 DFS event of 112, clinical relevance is limited. Although the DFS difference among patients with node-positive disease was numerically larger, NNT still was 56. Of note, some methodological problems need to be discussed, such as the comparator arm being underestimated with an expected 3-year DFS of $89.2 \%$. Most importantly, these data have to be analyzed in the concept of other adjuvant trials with HER2-targeted agents, such as the ALTTO trial, in which the addition of lapatinib failed to improve DFS. In the Extenet trial, 1 year of neratinib therapy up to 2 years after completion of (neo)adjuvant trastuzumab therapy was compared to placebo and rendered a significant $2 \%$-improvement of the primary study endpoint invasive-DFS
(iDFS) of 93.9 vs. $91.6 \%(\mathrm{HR} 0.67$ (95\%CI 0.5-0.91), $\mathrm{p}=0.0091$ ). However, these results have to be interpreted in light of added toxicities, particularly diarrhea (39.9 vs. $1.6 \%$ ). Overall, these data have to be discussed in view of toxicities, treatment costs, and - last but not least - in view of the hippocratic principle of 'primum non nocere'. In my view, personalized escalation of HER2-targeted therapy is warranted. Such a concept is evaluated in the postneoadjuvant KATHERINE study (NCT01772472) analyzing the effect of an addition of T-DM1 vs. continuation of trastuzumab among patients with residual invasive breast cancer after neoadjuvant therapy with chemotherapy and trastuzumab. Results are pending.

Müller: More than 10 years after the presentation of the large adjuvant studies with trastuzumab, the results of the AHINITY study illustrate that further improvement in HER2-postive breast cancer is possible. However, we also see that we have reached a very low level of recurrence in the overall cohort. Therefore, I think that low-risk patients certainly will not need dual blockade. However, the positive result of the adjuvant study also confirms the concept of neoadjuvant therapy which led to an approval of pertuzumab in the setting of early breast cancer. For high-risk patients (e.g., node positive and /or hormone receptor (HR)-negative) the (neo)adjuant treatment with dual blockade is the preferred option in my opinion. The results of several other studies presented also indicate that it might be possible to de-escalate chemotherapy which would be a great progress for our patients.

\section{Question 4: Is the Rise of CDK4/6 Inhibitors in ER+ Advanced Breast Cancer Still Continuing?}

Fehm: Yes, of course. George Sledge presented the results of the MONARCH-2 study. This double-blind phase III trial investigates the role of the CDK4/6 inhibitor abemaciclib in metastatic breast cancer as a second-line treatment. 669 patients were randomized to abemaciclib / fulvestrant $(\mathrm{F})$ and to placebo $(\mathrm{P})$ and fulvestrant $(\mathrm{F})$. The PFS was 16.4 months for abemaciclib / F and 9.3 months for P / F. The main side effects were diarrhea ( 86.4 vs. $24.7 \%$ ), neutropenia ( 46.0 vs. $4.0 \%)$ and nausea (45.1 vs. $22.99 \%)$. Therefore, in addition to palbociclib, there will be abemaciclib available for second-line treatment.

Liedtke: There is a large and solid body of evidence supporting the role of CDK4/6 inhibition among patients with metastatic HRpositive breast cancer. This year's ASCO has seen results of the MONARCH study program of the third CDK4/6 inhibitor abemaciclib that were in line with results presented for palbociblib and ribociclib. With regards to the latter 2 agents, results from 2 randomized trials failed to demonstrate an improvement of overall survival (OS) by the addition of CDK4/6 inhibition to endocrine therapy. First of all, methodological explanations, such as a limited number of patients, might be held responsible to these findings. However, these results may also reflect the difficulties of demonstrating an OS benefit in a patient population that will receive a 
large number of subsequent therapies that may obscure an OS effect. Therefore, these results have to be interpreted carefully particularly in face of current licensing and pricing discussions. Most importantly, these results do not question the efficacy of CDK4/6 inhibition among patients with metastatic HR-positive / HER2negative breast cancer.

Müller: Several studies for the 3 compounds currently approved or in development were presented. One of the open questions is the identification of potential biomarkers. Other important questions are the sequential use after failure of one CDK 4/6 inhibitor.

\section{Question 5: Was There Anything 'Practice Changing' to Influence Your Therapeutic Decisions Next Monday?}

Fehm: Not next Monday. But the study results of the OlympiaD trial will lead to a practice-changing treatment within the next months. Findings from this phase III trial demonstrated that heavily pretreated BRCA1/2-positive metastatic breast cancer patients who received olaparib as monotherapy have a significantly improved PFS compared to those patients treated with cytotoxic treatment including either capecitabine, vinorelbine, or erubilin. Olaparib was well tolerated. Less than $5 \%$ of the patients discontinued treatment. Therefore, in the near future, olaparib will be approved as monotherapy in pretreated BRCA1/2-positive metastatic breast cancer patients.

Liedtke: From my point of view, the results of the chemotherapy question of the adjuvant PlanB study, presented by Nadia Harbeck, may in fact influence therapy decisions right after ASCO. Prof. Harbeck showed the primary study endpoint of intention-to-treat DFS among 2,281 patients randomized to receive either 6 cycles of docetaxel / cyclophosphamide $\left(\mathrm{Doc}_{75} / \mathrm{Cyclo}_{600}\right.$ ) or 4 cycles of epirubicin / cyclophosphamide $\left(\mathrm{E}_{90} \mathrm{C}_{600} \times 4\right)$ followed by 4 cycles of docetaxel $\left(\operatorname{Doc}_{100} \times 4\right)$. Inclusion criteria comprised age $\leq 75$ years,
cM0 status, free margins (R0), and either $\mathrm{pN}+$ status or $\mathrm{pN} 0$ status with additional risk criteria ( $\mathrm{pT} \geq 2, \mathrm{G} 2-3$, elevated uPA/PAI-1 levels, HR negativity, age $\leq 35$ years). Chemotherapy was indicated (i) among all patients with HR-negative disease and (ii) among patients with HR-positive disease and either 0-3 metastatic axillary nodes and RS $\geq 11$ or $\geq 4$ metastatics axillary nodes. The study was planned as a non-inferiority trial and did reach its primary endpoint with an HR for DFS of 0.996 (95\%CI 0.77-1.29). These results suggest that this group of patients may well be spared longterm toxicities of anthracycline therapy (such as cardiotoxicity or myeloproliferative disease). These results may very well deserve immediate translation into daily practice.

Müller: I would try to offer olaparib to patients with a $B R C A$ mutation after failure of chemotherapy although this requires individual applications to the health insurance companies in Germany until approval for this indication.

\section{Participants}

Prof. Dr. med. Tanja Fehm

Frauenklinik

Universitätsklinikum Düsseldorf

Moorenstrasse 5, 40225 Düsseldorf, Germany

tanja.fehm@med.uni-duesseldorf.de

Prof. Dr. med. Cornelia Liedtke

Gynäkologie mit Brustzentrum

Charité-Universitätsmedizin Berlin

Campus Charité Mitte

Charitéplatz 1, 10117-Berlin, Germany

cornelia.liedtke@charite.de

Prof. Dr. med. Volkmar Müller

Klinik und Poliklinik für Gynäkologie

Universitätsklinikum Hamburg-Eppendorf

Martinistraße 52, 20246 Hamburg, Germany

vmueller@uke.de 